\section{Patient education for boys with functional urinary incontinence}

Functional urinary incontinence is a common disorder among children and adolescents and has a negative effect on quality of life. Training, involving behavioral, physical and cognitive elements, is now a popular method of treatment. Stauber and colleagues assessed the longterm efficacy of a patient education program which included cognitive-behavioural stress management training.

The study included 25 boys, aged between 8 and 12 years, who presented with urinary incontinence; 10 of these were assigned to the control group and 15 to the intervention group. The boys in the intervention group were educated with an emphasis on cognitive-behavioral stress management and coping skills and the boys in the control group received basic education without cognitivebehavioral training. The long- and short-term efficacy of the program, ability to cope and quality of life 6 months after commencing the program were investigated.

In both groups, 6 months after the start of the program, day-time wetting decreased and the ability to cope with common stressors increased, and night-time wetting decreased significantly in the intervention group only. The boys in the intervention group also experienced improved self-esteem and coping with illness-related stressors.

The authors conclude that children with low self-esteem and difficulty coping with illnessrelated stress would benefit from stress management training as part of their patient education behavioral program; however, given the small size of this study, further studies with larger sample sizes are warranted.

Original article Stauber T et al. (2007) Cognitivebehavioral stress management training for boys with functional urinary incontinence. J Pediatr Urol 3: 276-281

\section{Use of hydrophilic-coated catheters in patients with AUR and an abnormal urethra}

In most cases of acute urinary retention (AUR), bladder drainage is easily achieved by inserting a standard balloon retention catheter through the urethra. In the rare cases that routine catheterization is not possible due to abnormal urethral anatomy, more-invasive techniques (e.g. flexible cystourethroscopy) are required. New urethral catheters with hydrophilic, selflubricating coatings provide improved ease of entry and decreased discomfort; therefore, Mistry and colleagues investigated whether such catheters can be successfully used in male patients with AUR for whom standard catheterization fails.

The authors enrolled 44 participants with AUR. After failed attempts at insertion of a $12 \mathrm{~F}$ or $18 \mathrm{~F}$ standard catheter, subsequent insertion of hydrophilic catheters of the same diameters was performed. If successful insertion was achieved, a guidewire was threaded through the hydrophilic catheter and a balloon retention catheter was placed over the wire; if this complete procedure provided continuous bladder drainage, it was deemed successful.

The hydrophilic catheter was successfully placed, leading to relief of AUR, in $34(72 \%)$ of the 44 patients in the study. Of these, 30 (68.2\%) patients achieved long-term drainage by successful placement of the balloon catheter. The causes of complicated catheter placement included anterior urethral stricture, postoperative bladder neck contracture and urethral false passage.

The authors conclude that the use of hydrophilic-coated catheters might be a useful minimally-invasive approach for most patients with AUR and an abnormal urethra. Larger studies should be performed to identify those patients who will benefit most from this procedure.

Original article Mistry S et al. (2007) Use of hydrophiliccoated urethral catheters in management of acute urinary retention. Urology 70: 25-27

\section{Atorvastatin is ineffective for treating LUTS associated with BPH}

Research has suggested that the beneficial effects of atorvastatin on serum lipid parameters, such as reduced cholesterol and LDL, and its growth-inhibitory effects on epithelial cells, might be useful in the treatment of patients with benign prostatic hyperplasia (BPH). Mills and colleagues performed a phase II, doubleblind, randomized, placebo-controlled trial to test the efficacy of atorvastatin in patients with lower urinary tract symptoms (LUTS) caused by presumed BPH. 